\title{
Four and two-lepton signals of leptophilic gauge interactions at large colliders
}

\author{
Francisco del Aguila* ${ }^{*} a$, Mikael Chala ${ }^{b}$, José Santiago ${ }^{a, c}$ and Yasuhiro Yamamoto ${ }^{a}$ \\ ${ }^{a}$ CAFPE and Departamento de Física Teórica y del Cosmos, Universidad de Granada, \\ E-18071 Granada, Spain \\ ${ }^{b}$ DESY, Notkestrasse 85, 22607 Hamburg, Germany \\ ${ }^{c}$ Institute for Theoretical Physics, ETH Zürich, 8093 Zürich, Switzerland \\ E-mail: faguila@ugr.es, mikael.chala@desy.de, jsantiago@ugr.es, \\ yamayasulugr.es
}

\begin{abstract}
Many Standard Model extensions can contribute to four-lepton signals at large colliders. We review the particular case of leptophilic interactions eventually observable at the LHC and the ILC, paying special attention to the addition of a new vector boson coupled to muon minus tau lepton number, $Z_{\mu-\tau}^{\prime}$, and emphasizing the prospects at a very large hadron collider with $\sqrt{s}=$ $100 \mathrm{TeV}$. We also discuss in this case the new contribution to two-lepton (Drell-Yan) production when the new leptophilic interaction has a non-vanishing kinetic mixing with the SM.
\end{abstract}

Proceedings of the Corfu Summer Institute 2014

3-21 September 2014

Corfu, Greece

\footnotetext{
*Speaker.

${ }^{\dagger}$ We are grateful to the Corfu Summer Institute 2014 organizers for their hospitality.
} 


\section{Introduction}

The Standard Model (SM) has been completed with the discovery of the Brout-Englert-Higgs (BEH) boson [1] at the Large Hadron Collider (LHC) [2]. Besides, the outstanding performance of the machine and of the LHC experimental collaborations have also allowed to translate the nonobservation of any significative departure from the SM predictions into stringent limits on New Physics (NP). What, in particular, implies that if NP is going to show up at future runs, it must predict relatively small cross-sections into final states already scrutinized. Obviously, small crosssections can be always obtained banishing the NP scale to higher energies or coupling it to the SM weakly enough. The relevant question is if there is room left for new interactions near the $\mathrm{TeV}$ scale with effective couplings of at least Electro-Weak (EW) strength. What also brings to the question of whether NP can manifest at the International Linear Collider (ILC) if no departure from the SM can be established at the LHC.

Specific SM extensions can predict some new particles to be in practice invisible at the LHC even if their masses are near the EW scale. Indeed, the LHC discovery reach can be reduced allowing these heavy particles to decay into new channels with larger backgrounds, making in any case them wider and eventually undetectable. Their production, and then their detection, can be also made more difficult if they are constrained to be pair-produced by imposing the corresponding selection rule. There are many of these examples in the literature. For instance, stealth gluons [3] provide an example of the first case, and supersymmetric models with R-parity [4] of the second one. Often these properties reducing the LHC discovery potential are also retained at the ILC, not being then this $e^{+} e^{-}$machine an alternative for these NP searches. Although the actual sensitivity for a given process will depend on which circumstance plays a more important role, its lower Center of Mass Energy (CME) or its cleaner environment, and eventually the available beam polarization at this leptonic machine [5].

One can be more drastic, however, and assume that the new particles do not couple to quarks and gluons, nor to EW bosons, but only to SM leptons, at least to start with. Trying in this way to reduce as much as possible the LHC discovery reach, but not the ILC one. Nonetheless, even in this extreme scenario the LHC seems to be able of partially settling the question, whenever electrons (positrons) are not relevant. A detailed study of the LHC and the ILC potential for the discovery of these leptophilic interactions is presented in Ref. [6]. We summarize the main results presented there in Section 2, but concentrating on the particular case of an extra gauge boson coupling to muon minus tau Lepton Number (LN), $Z_{\mu-\tau}^{\prime}$ [7], as example. We then compare the LHC and ILC potentials with the potential of a Future Circular hadron Collider (FCC) with a CME $\sqrt{s}=100$ $\mathrm{TeV}[8]{ }^{1}$ in Section 3. Although such a leptophilic gauge boson tends to mix little with the SM $Z$ boson, the kinetic mixing can be large in generic models [11] and then, the $Z_{\mu-\tau}^{\prime}$ contribution to the Drell-Yan process, $q \bar{q} \rightarrow Z_{\mu-\tau}^{\prime} \rightarrow l \bar{l}$, could be eventually sizable at a large hadron collider. We discuss this scenario in Section 4 , finding that the limits on the complementary process at the LEP $2, e^{+} e^{-} \rightarrow Z_{\mu-\tau}^{\prime} \rightarrow l \bar{l}$, already constrain the kinetic mixing to be small for relatively low vector boson masses of few hundreds of GeV. However, Drell-Yan production at the LHC (FCC) provides the most stringent bound on such a mixing, especially for larger masses of the order of the TeV.

\footnotetext{
${ }^{1}$ Future Circular lepton Colliders have also deserved consideration [9], as well as a Large Hadron electron Collider (LHeC) [10] with different options.
} 


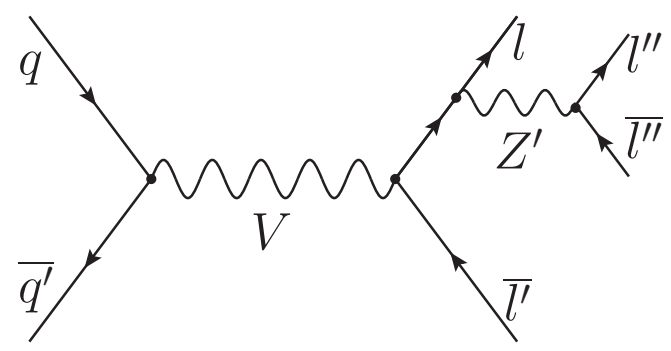

Figure 1: Leading diagram for $Z_{\mu-\tau}^{\prime}$ production at the LHC, for negligible kinetic mixing.

Thus, four-lepton production in Fig. 1 is the only leptophilic final state available for vanishing kinetic mixing but $q \bar{q} \rightarrow \gamma, Z, Z_{\mu-\tau}^{\prime} \rightarrow l \bar{l}$ allows to set quite strong limits on these interactions for large kinetic mixing. Final comments are collected in the Conclusions.

\section{Limits on $Z_{\mu-\tau}^{\prime}$ at the LHC and the ILC}

As we have recently argued in Ref. [6], the only leptophilic particle coupling to SM lepton pairs in a renormalizable way is a new neutral vector boson $Z^{\prime}$. If this boson was a scalar, it would have to be the component of a multiplet transforming as the BEH one or including a doublycharged component, too (see Ref. [12] for a detailed discussion of the lowest order couplings and production mechanisms for such a scalar). Thus, implying in either case that it would couple to SM gauge bosons with EW strength and hence, it would not be a leptophilic particle. (Although at the end, leptophilic additions are expected to partially lose their character because higher order effects may result in a small mixing between the different sectors of the theory, thus coupling to EW gauge bosons and to quarks, too, but with suppressed couplings.) Bosons with spin higher than 1 do not have renormalizable couplings to lepton pairs, either. The generic Lagrangian describing the corresponding vector boson interactions reads:

$$
\mathscr{L}_{Z^{\prime}}=-\left(g_{\mathrm{L}}^{\prime i j} \bar{L}_{i \mathrm{~L}} \gamma^{\mu} L_{j \mathrm{~L}}+g_{\mathrm{R}}^{\prime i j} \bar{l}_{i \mathrm{R}} \gamma^{\mu} l_{j \mathrm{R}}\right) Z_{\mu}^{\prime},
$$

where $g_{\mathrm{L}, \mathrm{R}}^{\prime i j}$ are arbitrary dimensionless couplings to the SM Left-Handed (LH) and Right-Handed (RH) lepton multiplets, $L_{i \mathrm{~L}}=\left(\begin{array}{c}v_{i \mathrm{~L}} \\ l_{i \mathrm{~L}}\end{array}\right)$ and $l_{i \mathrm{R}}$, respectively, with $i=e, \mu, \tau$ labeling the chargedlepton family. Even though we allow for arbitrary flavor and chiral interactions, the EW gauge symmetry remains unbroken because the new couplings of the LH charged leptons and their neutrino counterparts are equal. However, the Yukawa couplings giving masses to the charged leptons in the SM do not need to preserve such a hypothetical gauge symmetry. As a matter of fact, if a leptonic Yukawa term has to be invariant under the new symmetry, the scalar field coupling to the pair of LH and RH leptons must have a commensurate charge adding to zero with the lepton charges. Thus, in general, the existence of a new leptophilic interaction described by Eq. (2.1) requires non-minimal scenarios with a non-standard lepton mass generation and/or extra scalar multiplets for the model to be realistic.

An example of the first scenario, without extra light scalar multiplets and hence, without Yukawa terms involving leptons (because the SM BEH doublet must be a singlet under the new 
symmetry in order to quarks get SM masses), is the composite Higgs model with tau custodians in Ref. [13]. In this case, once the extra heavy vector bosons present in the model acquire a mass, lepton Yukawa couplings are generated at higher orders in perturbation theory.

A prime example of a model with an extra leptophilic interaction and possibly extra scalar doublets is the combination of muon minus tau $\mathrm{LN}, \mathrm{L}_{\mu}-\mathrm{L}_{\tau},{ }^{2}$ with diagonal couplings $g_{\mathrm{L}, \mathrm{R}}^{\prime \mu \mu, \tau \tau}$ given by the charges

$$
\begin{array}{l|cccc}
\text { Multiplet } & L_{\mathrm{L} \mu}=\left(\begin{array}{c}
v_{\mu \mathrm{L}} \\
\mu_{\mathrm{L}}
\end{array}\right) & \mu_{\mathrm{R}} & L_{\mathrm{L} \tau}=\left(\begin{array}{c}
v_{\tau \mathrm{L}} \\
\tau_{\mathrm{L}}
\end{array}\right) & \tau_{\mathrm{R}} \\
\hline \text { Charge } & 1 & 1 & -1
\end{array}
$$

times the gauge coupling constant $g^{\prime}$. This is the only gauge addition to the SM with vanishing couplings to electrons and anomaly free in the absence of extra fermions [7]. On the other hand, this model also allows for a good estimate (which can be also easily extrapolated [6]) of the LHC (and the ILC) potential for the discovery of leptophilic interactions. Its reach appears to be relatively reduced (in either case) because the two-lepton signal of the new resonating vector boson must in principle emerge from a four-lepton sample. This means that we must deal with relatively small cross-sections of few $\mathrm{fb}$ at the LHC for $\sqrt{s}=14 \mathrm{TeV}$ (and similarly at the ILC) and hence, that there is only enough sensitivity for an eventual discovery when the new gauge couplings are relatively large and/or the $Z^{\prime}$ masses are somewhat small. This then excludes possible electron signals if the limits from LEP 2 must be fulfilled [14]. Assuming universality, for these limits do not show a large flavor dependence, the corresponding $95 \%$ Confidence Level (C.L.) bounds read [15] (see also [16]):

$$
\frac{g_{\mathrm{L}, \mathrm{R}}^{\prime e e}}{M_{\mathrm{Z}^{\prime}}}<0.12,0.16 \mathrm{TeV}^{-1},
$$

being too stringent for observing a leptophilic vector boson in a four-lepton final state at the LHC (ILC). We assume that the model gauging $\mathrm{L}_{\mu}-\mathrm{L}_{\tau}$ also includes a SM singlet scalar giving a relatively large mass to the new gauge boson. The originally proposed realization addressed the possibility of a relatively light $Z_{\mu-\tau}^{\prime}$. In our case, the extra vector boson can be made arbitrarily heavy. We will advocate for the extra scalar doublets transforming non-trivially under the new gauge symmetry only to generate an appreciably mass mixing with the SM gauge bosons, when needed. Neutrino masses are very tiny and we assumed they are generated at higher orders in a non-minimal scenario. In any case, in the following the parameters used to fix the model in the Monte Carlo simulations shall be only the new gauge coupling $g^{\prime}$ and the heavy vector boson mass $M_{Z_{\mu-\tau}^{\prime}}$; while the total width writes:

$$
\Gamma_{Z_{\mu-\tau}^{\prime}}=\frac{g^{\prime 2}}{4 \pi} M_{Z_{\mu-\tau}^{\prime}}
$$

where we neglect any $Z_{\mu-\tau}^{\prime}$ decay into new scalar pairs.

The LHC and ILC limits on a generic leptophilic vector boson only coupling to muons and taus are throughly discussed in Ref. [6]. Let us review here the case of the $Z_{\mu-\tau}^{\prime}$ above. In general, such

\footnotetext{
${ }^{2}$ In order to give a large enough mass to the new gauge boson, the model must also include at least one extra scalar field transforming trivially under the SM gauge symmetry but not under $\mathrm{L}_{\mu}-\mathrm{L}_{\tau}$.
} 

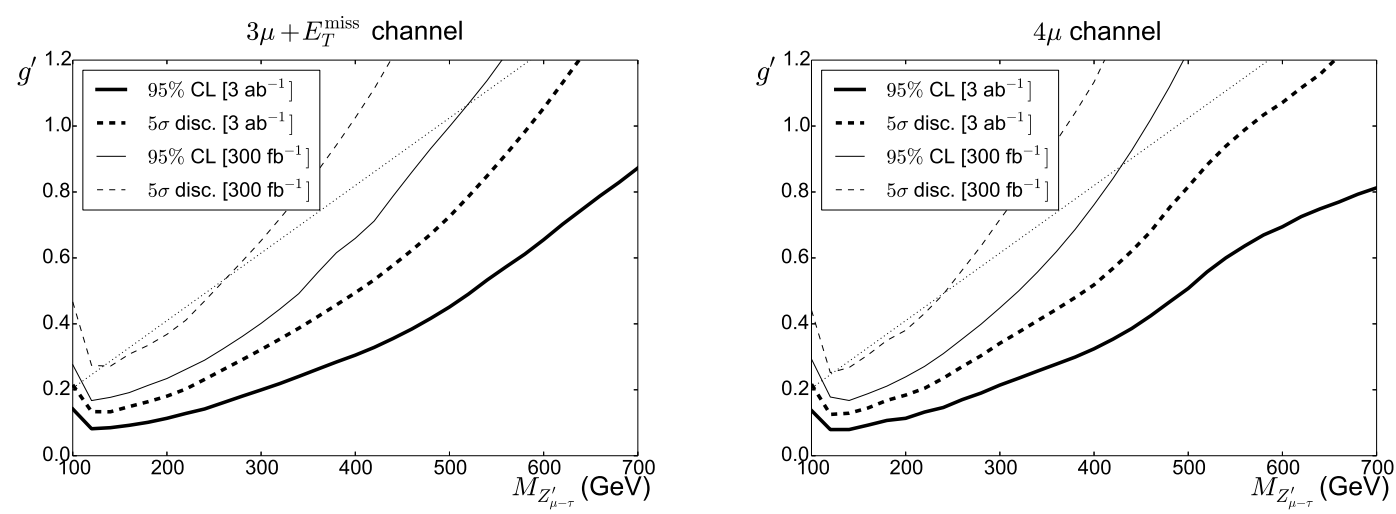

Figure 2: Discovery and exclusion limits for $Z_{\mu-\tau}^{\prime}$ as a function of its mass at the LHC with $\sqrt{s}=14 \mathrm{TeV}$. On the left (right) we plot the bounds obtained from $3 \mu$ plus missing energy $(4 \mu)$ events. We also draw the bounds from neutrino trident production (straight line), for comparison (see the text for details).

a vector boson will show up in the four-lepton final states. As a matter of fact, the best limits at the LHC can be generally derived from the $3 \mu$ plus missing energy events. Although for this model the bounds from $4 \mu$ are somewhat similar. In Fig. 2 we plot the corresponding discovery and exclusion bounds for the $3 \mu$ plus missing energy (left) and $4 \mu$ (right) samples. As explained in Ref. [6], these curves can be obtained from the plots in this reference multiplying by the appropriate factors. In particular, the exclusion bound can be read from Fig. 3 with $\xi \equiv g_{\mathrm{R}}^{\prime} / g_{\mathrm{L}}^{\prime}=1$ there multiplying the corresponding coupling constant for a given $Z^{\prime}$ mass by $\sqrt{2}$, to take into account the effect of the extra vector boson decay channel into taus. The event generation (codes used) and selection (applied cuts) are described in that reference, too. For comparison, we also show the bound from the neutrino trident production (straight line) [17], taking into account CHARM-II and CCFR data.

Whereas only signals involving muons can emerge at the LHC for the $Z_{\mu-\tau}^{\prime}$ cross-sections at hand, as pointed out in Ref. [6], the ILC could observe the mixed channels with tau leptons. Thus, in Fig. 3 we show the corresponding discovery and exclusion limits but for $4 \mu$ and $2 \mu 2 \tau$ events, respectively. Again, the bounds can be read from that reference. In particular, the limits obtained from $4 \mu$ events can be read from Fig. 7 (right) there multiplying the corresponding coupling constant for a given $Z^{\prime}$ mass by $\sqrt{2}$, to account for the extra decay channel into taus. Details on the event generation and selection cuts are given in the same reference.

As emphasized in Ref. [6], the LHC can be sensitive to a $Z_{\mu-\tau}^{\prime}$ with a mass up to $\sim 1 \mathrm{TeV}$ for $g^{\prime}$ of order 1 ; while the ILC is sensitive to $Z_{\mu-\tau}^{\prime}$ masses below $300 \mathrm{GeV}$ but it can eventually allow for a direct measurement of the couplings to tau leptons.

\section{Limits on $Z_{\mu-\tau}^{\prime}$ at a future hadron collider with $\sqrt{s}=100 \mathrm{TeV}$}

As already pointed out, the LHC and the ILC will improve the limits on a new leptophilic $Z^{\prime}$ unmixed with the SM, but for relatively large coupling constants and relatively small vector boson masses. Their reach will be appreciably enlarged at a larger hadron collider with a CME of $\sqrt{s}=$ $100 \mathrm{TeV}$, however. Similarly as for the LHC in the previous section, in Fig. 4 we plot the discovery and exclusion bounds for the $3 \mu$ plus missing energy (left) and $4 \mu$ (right) samples. The codes used 

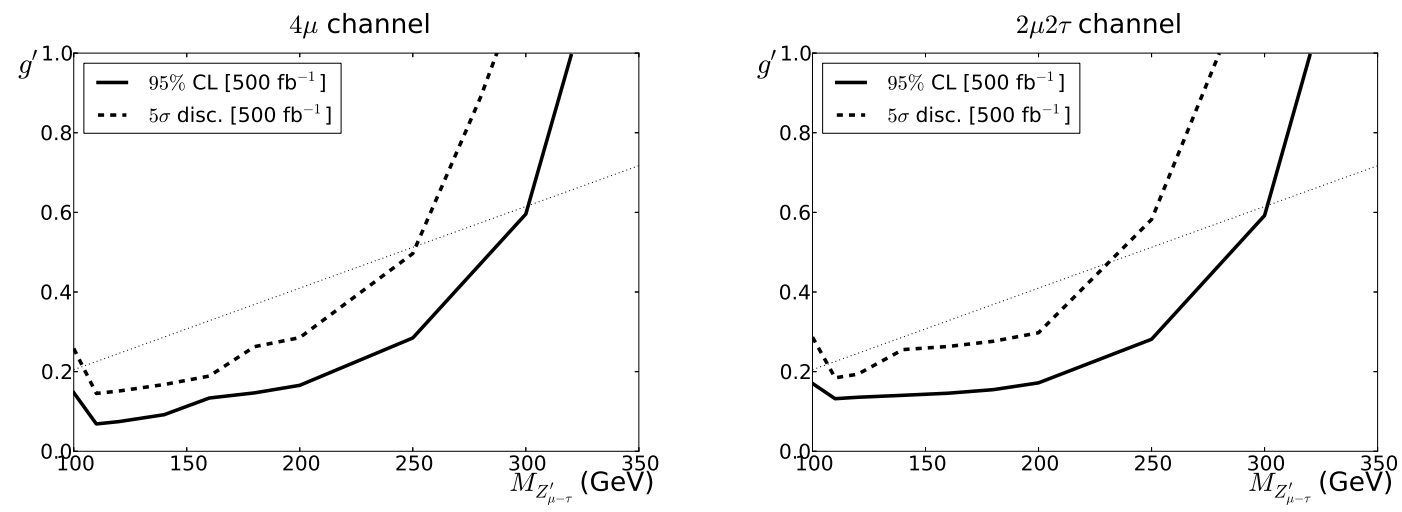

Figure 3: Discovery and exclusion limits for $Z_{\mu-\tau}^{\prime}$ as a function of its mass at the ILC with $\sqrt{s}=500 \mathrm{GeV}$. On the left (right) we plot the bounds obtained from $4 \mu(2 \mu 2 \tau)$ events. The constraint from neutrino trident production (straight line) is also shown for comparison.
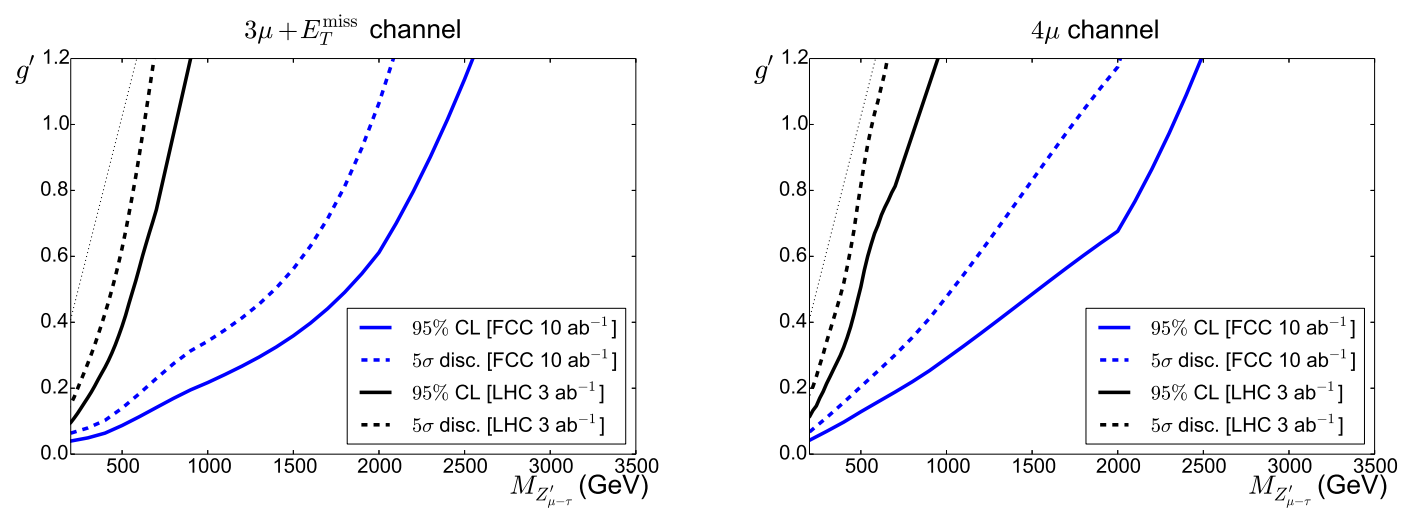

Figure 4: Discovery and exclusion limits for $Z_{\mu-\tau}^{\prime}$ as a function of its mass at the future hadron collider (FCC) with $\sqrt{s}=100 \mathrm{TeV}$ (thick curves). On the left (right) we plot the bounds obtained from $3 \mu$ plus missing energy $(4 \mu)$ events. We also draw present bounds from neutrino trident production (straight line) and the LHC limits for $\sqrt{s}=14 \mathrm{TeV}$ (thin curves), for comparison.

for the event generation are the same as in Ref. [6]. The selection cuts applied to the $3 \mu$ plus missing energy sample must be optimized, however, to obtain similar limits to those derived from the $4 \mu$ channel. This is necessary to get rid of the background events in that case, too. Thus, we increase the basic cut on the muon transverse momentum in Table 1 in the previous reference from 50 to $100 \mathrm{GeV}$; and the cuts on the missing transverse energy and on the transverse mass from 100 to $150 \mathrm{GeV}$ and from 110 to $140 \mathrm{GeV}$, respectively. As a result, the FCC bounds on the gauge coupling improve by almost a factor of 5 those at the LHC for low $Z_{\mu-\tau}^{\prime}$ masses; whereas its mass reach is near $2.5 \mathrm{TeV}$ for $g^{\prime} \sim 1$.

\section{4. $Z_{\mu-\tau}^{\prime}$ kinetic mixing with the SM and di-lepton versus four-lepton signals of a leptophilic vector boson}

There is a wide literature on the implications of the kinetic mixing between abelian gauge 
factors [18] in SM gauge extensions [19]. We will follow Ref. [11] because it emphasizes our main observation in this section concerning the size of the kinetic mixing of an extra abelian interaction with the SM: the effective mass mixing between a new $Z^{\prime}$ and the SM $Z$ boson must be rather small, as required by present data, but the kinetic mixing can be relatively large because it only enters alone in the $Z^{\prime}$ interactions. Indeed, all deviations from the SM predictions for processes involving only SM particles and, in particular, testing the $Z$ boson interactions are proportional to the former and hence, it must be tiny (below the per mille level) because no significant departure from the SM has been observed. While a non-vanishing kinetic mixing modifies the $Z^{\prime}$ current adding an extra term to it, linear in the mixing and proportional to the SM hypercharge, allowing to constrain its size in an independent way in processes exchanging the new vector boson. In the case of the $Z_{\mu-\tau}^{\prime}$ it means, for example, that although this gauge boson is leptophilic to start with, it also couples to quarks because they have non-zero hypercharge but with couplings suppressed by the kinetic mixing. The relevant question is then how small this mixing must be on phenomenological grounds.

Let us be quantitative. Following (the notation of) Ref. [11] the effective mass mixing, $\xi$, rotating the current eigenstate gauge bosons to the heavy mass eigenstate basis, can be written (note that we used $\xi$ before to specify the RH $Z^{\prime}$ couplings in Ref. [6]):

$$
\tan 2 \xi \approx-2 \frac{\delta M^{2}+\sin \chi s_{W} M_{Z}^{2}}{M_{Z_{\mu-\tau}^{\prime}}^{2}}
$$

where $\delta M^{2}$ is the mass term mixing the gauge boson current eigenstates and $\sin \chi$ the corresponding kinetic mixing (and $s_{W}$ the sine of the EW mixing angle),

$$
\mathscr{L}_{\text {mixing }} \approx \delta M^{2} Z_{\mu-\tau}^{\prime \sigma} Z_{\sigma}-\frac{\sin \chi}{2} Z_{\mu-\tau}^{\prime \sigma v} B_{\sigma v}
$$

We only keep the leading terms in the limit of $\xi$ and $\chi$ small (fields are in the physical basis). We will concentrate on the case $\xi \ll \chi$, besides, which can be the result of a large mass hierarchy $M_{Z}^{2} \ll M_{Z_{\mu-\tau}^{\prime}}^{2}$, as we have formally required when deriving Eq. (4.1), or of a partial cancellation of the two terms in the numerator of this equation, or of both. A large $Z_{\mu-\tau}^{\prime}$ mass can be generated by a large vacuum expectation value (vev) of a scalar field transforming trivially under the SM gauge symmetry; whereas the mass mixing cancelling the kinetic term can be obtained from a scalar multiplet transforming non-trivially under both the SM and the extra abelian gauge symmetry and developing a vev of the appropriate size. ${ }^{3}$

The new gauge boson interactions with the SM fermions are described by the Lagrangian

$$
\begin{aligned}
\mathscr{L}_{Z_{\mu-\tau}^{\prime}} & \approx-\left(g^{\prime} J_{\sigma}^{Z_{\mu-\tau}^{\prime}}-\sin \chi \frac{e}{c_{W}} J_{\sigma}^{Y}\right) Z_{\mu-\tau}^{\prime \sigma} \\
& =-\left[\left(g^{\prime}+\sin \chi \frac{e}{2 c_{W}}\right)\left(\bar{v}_{\mu \mathrm{L}} \gamma_{\sigma} v_{\mu \mathrm{L}}+\bar{\mu}_{\mathrm{L}} \gamma_{\sigma} \mu_{\mathrm{L}}\right)+\left(g^{\prime}+\sin \chi \frac{e}{c_{W}}\right) \bar{\mu}_{\mathrm{R}} \gamma_{\sigma} \mu_{\mathrm{R}}\right.
\end{aligned}
$$

\footnotetext{
${ }^{3}$ Many phenomenological analyses neglect kinetic mixing effects, as, for instance, when determining the $Z Z^{\prime}$ mixing at the LHC [20]; or the mass mixing generated by the vev of scalar fields transforming non-trivially under both abelian factors, as in Higgs-portal studies [21]. Although the kinetic mixing can be large and the effective mass mixing small in specific models [22].
} 

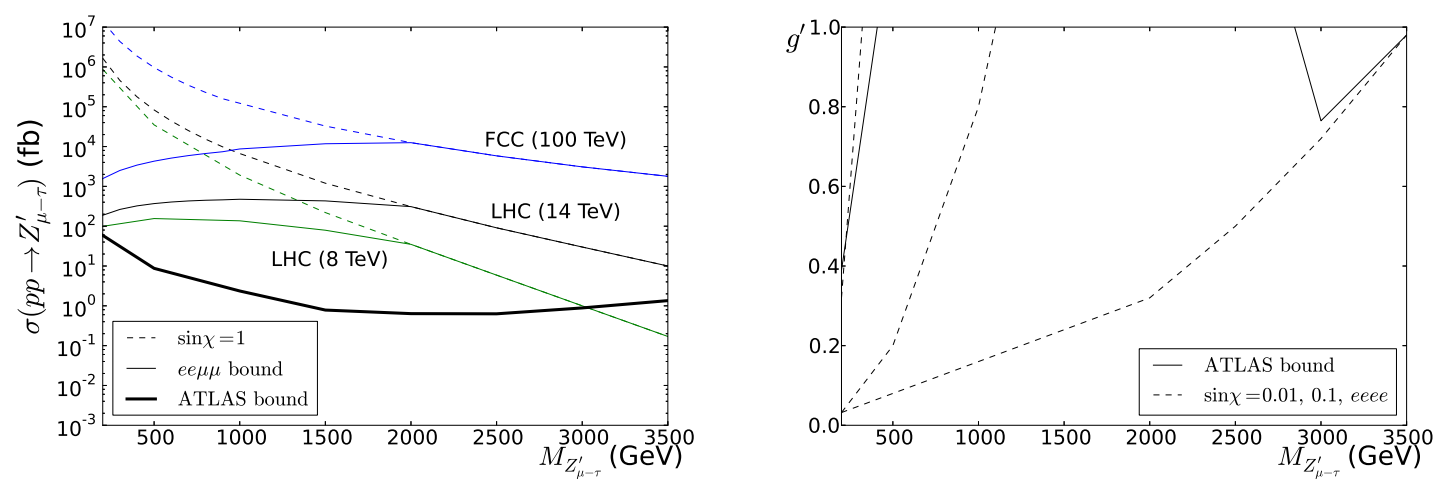

Figure 5: On the left we plot the total cross-section for $p p \rightarrow Z_{\mu-\tau}^{\prime}$ for different values of $\sin \chi$ (see the text) as a function of the new gauge boson mass at the LHC and at the FCC. On the right we show in the $g^{\prime}-M_{Z_{\mu-\tau}^{\prime}}$ plane the excluded (upper) regions by the LEP 2 and ATLAS bounds (see the text).

$$
\begin{aligned}
& +\left(-g^{\prime}+\sin \chi \frac{e}{2 c_{W}}\right)\left(\bar{v}_{\tau \mathrm{L}} \gamma_{\sigma} \nu_{\tau \mathrm{L}}+\bar{\tau}_{\mathrm{L}} \gamma_{\sigma} \tau_{\mathrm{L}}\right)+\left(-g^{\prime}+\sin \chi \frac{e}{c_{W}}\right) \bar{\tau}_{\mathrm{R}} \gamma_{\sigma} \tau_{\mathrm{R}} \\
& +\sin \chi \frac{e}{2 c_{W}}\left(\bar{v}_{e \mathrm{~L}} \gamma_{\sigma} v_{e \mathrm{~L}}+\bar{e}_{\mathrm{L}} \gamma_{\sigma} e_{\mathrm{L}}\right)+\sin \chi \frac{e}{c_{W}} \bar{e}_{\mathrm{R}} \gamma_{\sigma} e_{\mathrm{R}} \\
& \left.-\sin \chi \frac{e}{6 c_{W}}\left(\bar{u}_{\mathrm{L}} \gamma_{\sigma} u_{\mathrm{L}}+\bar{d}_{\mathrm{L}} \gamma_{\sigma} d_{\mathrm{L}}\right)-\sin \chi \frac{2 e}{3 c_{W}} \bar{u}_{\mathrm{R}} \gamma_{\sigma} u_{\mathrm{R}}+\sin \chi \frac{e}{3 c_{W}} \bar{d}_{\mathrm{R}} \gamma_{\sigma} d_{\mathrm{R}}+\ldots\right] Z_{\mu-\tau}^{\prime \sigma},
\end{aligned}
$$

where the dots stand for the same quark couplings as for the first family but for the other two quark generations. Thus, a non-vanishing kinetic mixing modifies the $Z_{\mu-\tau}^{\prime}$ current, adding an extra contribution linear in $\sin \chi$ and proportional to the SM hypercharge, $Y=Q-T_{3}$, with $Q$ the electric charge and $T_{3}$ the third component of isospin. Therefore, it adds $Z_{\mu-\tau}^{\prime}$ couplings to electrons and to quarks, implying a new contribution to Drell-Yan production at the LEP $2, e^{+} e^{-} \rightarrow Z_{\mu-\tau}^{\prime} \rightarrow l \bar{l}$, and at the LHC, $q \bar{q} \rightarrow Z_{\mu-\tau}^{\prime} \rightarrow l \bar{l}$. In that case, present limits on the former also restrict the allowed size of the kinetic mixing because no departure from the SM predictions has been observed but only for relatively low $Z_{\mu-\tau}^{\prime}$ masses. In fact, the limits in Eq. (2.3) translate into (using the couplings in Eq. (4.3)):

$$
\left.\frac{|\sin \chi| g^{\prime}}{M_{Z_{\mu-\tau}^{\prime}}^{2}}<0.07 \mathrm{TeV}^{-2} \quad(\text { eе } \mu \mu), \quad \frac{|\sin \chi|}{M_{Z_{\mu-\tau}^{\prime}}}<0.45 \mathrm{TeV}^{-1} \quad \text { (ееее }\right) .
$$

These bounds apply to the RH operators, which provide the most stringent limits due to the chirality dependence of the hypercharge couplings. The leptonic couplings in Eq. (4.3) are also nonuniversal, but lepton universality was assumed in order to derive Eq. (2.3). In this sense, although it is a rather good approximation, the quantitative analysis is approximate. We also neglect higher order terms in $\sin \chi$. In any case, although these bounds result in small gauge couplings to quarks for small gauge boson masses, the direct $Z_{\mu-\tau}^{\prime}$ production cross-section can be still sizable at the LHC if the LEP 2 limits on $\sin \chi$ are saturated. As a matter of fact, these bounds become unrestrictive for large vector boson masses. To be quantitative, we plot in Fig. 5 (left) the total new vector boson production cross-section $\sigma\left(p p \rightarrow Z_{\mu-\tau}^{\prime}\right)$, which is proportional to $|\sin \chi|^{2}$, at the LHC and the FCC for $\sin \chi=1$ (dashed lines) and for $\sin \chi$ saturating the first inequality in Eq. (4.4) with $g^{\prime}=0.25$ (solid lines). (Obviously, we always require $|\sin \chi| \leq 1$.) The second inequality, which 
is independent of $g^{\prime}$, is less restrictive in this case, and the corresponding curve lies in between the other two lines plotted. As it is apparent, the exclusion of two-lepton cross-sections below the $\mathrm{fb}$ by the LHC and the FCC will provide quite stringent limits on the kinetic mixing as well as on the mass and coupling of a $Z_{\mu-\tau}^{\prime}$. As a matter of fact, the limit reported by the ATLAS Collaboration at the LHC with $\sqrt{s}=8 \mathrm{TeV}$ on the production cross-section of a new neutral gauge boson decaying into two muons $\sigma\left(p p \rightarrow Z^{\prime}\right) \mathrm{Br}\left(Z^{\prime} \rightarrow \mu^{+} \mu^{-}\right)$[23] is already more restrictive, for a generic $Z_{\mu-\tau}^{\prime} \rightarrow \mu^{+} \mu^{-}$branching ratio of $1 / 3$, than the LEP 2 one, as shown in the same picture (lowest curve). It must be again emphasized that this figure is only for illustration, because we assumed a small enough kinetic mixing when deriving the Lagrangian in Eq. (4.3), for instance, but draw the curves for arbitrary $\sin \chi$. Similarly, the gauge boson branching ratio into muons may be smaller than $1 / 3$ for non-zero kinetic mixing. In fact, the ATLAS bound defines a band depending on this branching ratio (neglecting fermion masses):

$$
\operatorname{Br}\left(Z_{\mu-\tau}^{\prime} \rightarrow \mu^{+} \mu^{-}\right)=\frac{1+1.5 x+0.625 x^{2}}{3+5 x^{2}}, \quad x=\frac{\sin \chi}{g^{\prime}} \frac{e}{c_{W}},
$$

which ranges from 0.01 to 0.45 . With the same warning, on the right panel we show the excluded (upper) regions in the $g^{\prime}-M_{Z_{\mu-\tau}^{\prime}}$ plane by the first LEP 2 inequality in Eq. (4.4) for $\sin \chi$ saturating the second inequality in the same equation (lowest dashed line) and for two other illustrative values of $\sin \chi=0.1,0.01$. The smaller the kinetic mixing is, the larger the allowed region in the $g^{\prime}-M_{Z_{\mu-\tau}^{\prime}}$ plane is. We also draw the ATLAS Collaboration limit (solid line) on muon-pair production mediated by a new neutral gauge boson [23] saturating the first inequality of Eq. (4.4), for illustration. Obviously, the observation of a $Z_{\mu-\tau}^{\prime}$ resonance in four and two-muon events would allow for a determination of both parameters, $g^{\prime}$ and $\sin \chi$, once fixed the gauge boson mass.

The previous discussion is based on phenomenological grounds and hence, a final comment on the expected size of the kinetic mixing is in order. As the studied model is renormalizable, quantum corrections can be actually calculated, being in general the renormalization of the kinetic mixing quite small and below the per mille level. Thus, a relatively large mixing as the one saturating Eq. (4.4) requires a similar value at the matching scale. Therefore, although Drell-Yan production overcomes four-lepton production except for rather small kinetic mixing, the latter provides the most stringent model-independent limits for gauge boson masses below a few $\mathrm{TeV}$.

\section{Conclusions}

We have reviewed the LHC and ILC discovery potential for leptophilic interactions, which are characterized by their negligible coupling to quarks and EW bosons [6]. In particular, we have discussed in detail the case of an extra gauge boson coupling to $\mathrm{L}_{\mu}-\mathrm{L}_{\tau}$, as it also provides a good estimate of the reach of these machines. If it only couples appreciably to muons and taus, as assumed, it can be only observed as a resonance in the di-muon distribution of four-lepton final states. Then, the corresponding Monte Carlo simulation shows that the best LHC (ILC) limits can be derived using $3 \mu$ plus missing energy $(4 \mu)$ events. We have found that the LHC can be sensitive up to $Z_{\mu-\tau}^{\prime}$ masses near the $\mathrm{TeV}$ for $g^{\prime}$ of order 1 . The ILC can be only sensitive to $Z_{\mu-\tau}^{\prime}$ masses below $300 \mathrm{GeV}$ but it could eventually provide a direct measurement of the vector boson couplings to tau leptons. Similarly, we have studied the corresponding bounds for the FCC, finding that it is 
sensitive to gauge boson masses of $\sim 2.5 \mathrm{TeV}$ for $g^{\prime} \sim 1$. and to gauge couplings almost 5 times smaller than at the LHC for lower $Z_{\mu-\tau}^{\prime}$ masses.

All these results, however, assume a vanishing kinetic mixing of the new vector boson with the SM (hypercharge). Otherwise, the current coupling to the extra vector boson gets a new term proportional to the kinetic mixing and to the hypercharge and hence, the new vector boson also couples to electrons and to quarks but with couplings suppressed by the kinetic mixing and ratios fixed by the SM hypercharges. What implies new contributions to Drell-Yan production mediated by the new gauge boson, improving appreciably the LHC and ILC discovery potential due to their sensitivity to lepton pair production, $p p \rightarrow \gamma, Z, Z^{\prime} \rightarrow l \bar{l}$ and $e^{+} e^{-} \rightarrow \gamma, Z, Z^{\prime} \rightarrow l \bar{l}$, respectively. In fact, the new contributions are in both cases proportional to the kinetic mixing square. Limits from LEP 2 force this mixing to be at most of few per cent for relatively low $Z_{\mu-\tau}^{\prime}$ masses $(\sim 300 \mathrm{GeV})$ but leave it essentially unconstrained for gauge boson masses of the order of few $\mathrm{TeV}$. In contrast, the LHC data already provide the strongest bounds on this mixing, reaching the per mille level if no departure from the SM is observed with the full expected energy and luminosity. This limit could be eventually improved by another order of magnitude at the FCC.

Few comments are in order to conclude. This study enforces the need to give experimental results specifying the flavor in the sampling, and to provide limits as model independent as possible on the corresponding cross-sections [23]. Although the kinetic mixing may receive small quantum corrections, its size can be large at low energy due to the matching with a more fundamental theory at high scale, hence making the phenomenological analysis compulsory. Finally, the SM extension with an extra gauge boson coupled to $\mathrm{L}_{\mu}-\mathrm{L}_{\tau}$ has been recently invoked to explain small discrepancies in Higgs and $B$ decays [24], although the proposed models include extra matter and assumptions beyond the phenomenological analysis performed here. Leptophilic interactions have been also considered to produced and annihilate dark matter [25]. In any case, many approaches corner NP in the heavier families, especially when dealing with the lepton sector [26].

\section{Acknowledgements}

This work has been supported in part by the European Commission through the contract PITNGA-2012-316704 (HIGGSTOOLS), by the Ministry of Economy and Competitiveness (MINECO), under grant numbers FPA2010-17915 and FPA2013-47836-C3-1,2-P, and by the Junta de Andalucía grants FQM 101 and FQM 6552.

\section{References}

[1] F. Englert and R. Brout, Phys. Rev. Lett. 13 (1964) 321; P. W. Higgs, Phys. Rev. Lett. 13 (1964) 508.

[2] G. Aad et al. [ATLAS Collaboration], Phys. Lett. B 716 (2012) 1 [arXiv:1207.7214 [hep-ex]]; S. Chatrchyan et al. [CMS Collaboration], Phys. Lett. B 716 (2012) 30 [arXiv:1207.7235 [hep-ex]].

[3] R. Barcelo, A. Carmona, M. Masip and J. Santiago, Phys. Lett. B 707 (2012) 88 [arXiv:1106.4054 [hep-ph]]; see for related work and further references: J. A. Aguilar-Saavedra, D. Amidei, A. Juste and M. Perez-Victoria, arXiv:1406.1798 [hep-ph].

[4] G. R. Farrar and P. Fayet, Phys. Lett. B 76 (1978) 575; see for a recent review and further references: R. N. Mohapatra, arXiv:1503.06478 [hep-ph]. 
[5] H. Baer, T. Barklow, K. Fujii, Y. Gao, A. Hoang, S. Kanemura, J. List and H. E. Logan et al., arXiv:1306.6352 [hep-ph], and references there in.

[6] F. del Aguila, M. Chala, J. Santiago and Y. Yamamoto, JHEP 1503 (2015) 059 [arXiv:1411.7394 [hep-ph]].

[7] R. Foot, X. G. He, H. Lew and R. R. Volkas, Phys. Rev. D 50 (1994) 4571 [hep-ph/9401250]; and references there in.

[8] A. Ball et al., Future Circular Collider Study, Hadron Collider Parameters, Tech. Rep. FCC-ACC-SPC-0001, CERN, Geneva, 2014.

[9] J. Wenninger et al., Future Circular Collider Study, Lepton Collider Parameters, Tech. Rep. FCC-ACC-SPC-0003 (Rev. 2.0), CERN, Geneva, 2014.

[10] J. L. Abelleira Fernandez et al. [LHeC Study Group Collaboration], J. Phys. G 39 (2012) 075001 [arXiv:1206.2913 [physics.acc-ph]].

[11] K. S. Babu, C. F. Kolda and J. March-Russell, Phys. Rev. D 57 (1998) 6788 [hep-ph/9710441].

[12] F. del Aguila, M. Chala, A. Santamaria and J. Wudka, Phys. Lett. B 725 (2013) 310 [arXiv:1305.3904 [hep-ph]]; EPJ Web Conf. 60 (2013) 17002 [arXiv:1307.0510 [hep-ph]]; F. del Aguila and M. Chala, JHEP 1403 (2014) 027 [arXiv:1311.1510 [hep-ph]]; F. del Aguila, M. Chala, A. Santamaria and J. Wudka, Acta Phys. Polon. B 44 (2013) 11, 2139 [arXiv:1311.2950 [hep-ph]].

[13] F. del Aguila, A. Carmona and J. Santiago, JHEP 1008 (2010) 127 [arXiv:1001.5151 [hep-ph]]; Phys. Lett. B 695 (2011) 449 [arXiv:1007.4206 [hep-ph]].

[14] S. Schael et al. [ALEPH and DELPHI and L3 and OPAL and LEP Electroweak Collaborations], Phys. Rept. 532 (2013) 119 [arXiv:1302.3415 [hep-ex]].

[15] J. de Blas, EPJ Web Conf. 60 (2013) 19008 [arXiv:1307.6173 [hep-ph]].

[16] F. del Aguila, J. de Blas and M. Perez-Victoria, JHEP 1009 (2010) 033 [arXiv:1005.3998 [hep-ph]]; F. del Aguila and J. de Blas, Fortsch. Phys. 59 (2011) 1036 [arXiv:1105.6103 [hep-ph]]; J. de Blas, M. Chala and J. Santiago, Phys. Rev. D 88 (2013) 095011 [arXiv:1307.5068 [hep-ph]].

[17] W. Altmannshofer, S. Gori, M. Pospelov and I. Yavin, Phys. Rev. D 89 (2014) 095033 [arXiv:1403.1269 [hep-ph]]; Phys. Rev. Lett. 113 (2014) 091801 [arXiv:1406.2332 [hep-ph]].

[18] B. Holdom, Phys. Lett. B 166 (1986) 196.

[19] F. del Aguila, G. A. Blair, M. Daniel and G. G. Ross, Nucl. Phys. B 272 (1986) 413; F. del Aguila, G. D. Coughlan and M. Quiros, Nucl. Phys. B 307 (1988) 633 [Erratum-ibid. B 312 (1989) 751]; R. Foot and X. G. He, Phys. Lett. B 267 (1991) 509; C. D. Carone and H. Murayama, Phys. Rev. D 52 (1995) 484 [hep-ph/9501220]; F. del Aguila, M. Masip and M. Perez-Victoria, Nucl. Phys. B 456 (1995) 531 [hep-ph/9507455]; see for a review and further references: P. Langacker, Rev. Mod. Phys. 81 (2009) 1199 [arXiv:0801.1345 [hep-ph]]; K. A. Olive et al. [Particle Data Group Collaboration], Chin. Phys. C 38 (2014) 090001.

[20] V. V. Andreev, P. Osland and A. A. Pankov, Phys. Rev. D 90 (2014) 055025 [arXiv:1406.6776 [hep-ph]].

[21] S. Y. Choi, C. Englert and P. M. Zerwas, Eur. Phys. J. C 73 (2013) 2643 [arXiv:1308.5784 [hep-ph]].

[22] M. Hirsch, W. Porod, L. Reichert and F. Staub, Phys. Rev. D 86 (2012) 093018 [arXiv:1206.3516 [hep-ph]]; see also: F. del Aguila, Acta Phys. Polon. B 25 (1994) 1317 [hep-ph/9404323]. 
[23] G. Aad et al. [ATLAS Collaboration], Phys. Rev. D 90 (2014) 5, 052005 [arXiv:1405.4123 [hep-ex]]; arXiv:1502.07177 [hep-ex].

[24] J. Heeck, M. Holthausen, W. Rodejohann and Y. Shimizu, arXiv:1412.3671 [hep-ph]; A. Crivellin, G. D’Ambrosio and J. Heeck, arXiv:1501.00993 [hep-ph]; Phys. Rev. D 91 (2015) 7, 075006 [arXiv:1503.03477 [hep-ph]].

[25] N. F. Bell, Y. Cai, R. K. Leane and A. D. Medina, Phys. Rev. D 90 (2014) 3, 035027 [arXiv:1407.3001 [hep-ph]].

[26] R. Alonso, M. B. Gavela, G. Isidori and L. Maiani, JHEP 1311 (2013) 187 [arXiv:1306.5927 [hep-ph]]. 